\title{
New observer-based control design for mismatched uncertain systems with time-delay
}

\author{
VAN VAN HUYNH
}

\begin{abstract}
In this paper, the state estimation problem for a class of mismatched uncertain time-delay systems is addressed. The estimation uses observer-based control techniques. The mismatched uncertain time-delay systems investigated in this study include mismatched parameter uncertainties in the state matrix and in the delayed state matrix. First, based on a new lemma with appropriately choosing Lyapunov functional, new results for stabilization of mismatched uncertain time-delay systems are provided on the basis of a linear matrix inequality (LMI) framework and the asymptotic convergence properties for the estimation error is ensured. Second, the control and observer gains are given from single LMI feasible solution which can overcome the drawback of the bilinear matrix inequalities approach often reported in the literature. Finally, a numerical example is used to demonstrate the efficacy of the proposed method.
\end{abstract}

Key words: observer-based control, time-delay systems, linear matrix inequality (LMI), mismatched uncertain systems.

\section{Introduction}

The states of a system are not always measurable in many control systems and applications due to unavailability of sensors and owing to infeasibility of measurement schemes. In this situation, there are two approaches in designing the control scheme for stabilization. One is to use the output-based controllers, such as static gain and dynamic compensator types [1-2]. The other is to utilize state observers to provide an estimate of the unmeasured states [3-16]. The first approach is achieved under assumption that the norm of un-measurement states must be bounded by a constant value $\left(\left\|T_{1} x\right\| \leqslant \mu_{1}\right.$ or $\|z\| \leqslant q_{2}$ ) [1-2]. In practical cases, these conditions are difficult to meet. Therefore, the design of observer-based control is very important and has been attracting the attention for researchers in recent years [3-16]. In [3], the robust observer-based control is proposed for a class of linear systems without input uncertainty. A class of uncertain systems with disturbance input is considered in [4]. In [5], based on linear matrix inequalities (LMI) the controller gain and the observer gain are designed to stabilize a

The Author is with Modeling Evolutionary Algorithms Simulation and Artificial Intelligence, Faculty of Electrical and Electronics Engineering, Ton Duc Thang University, Ho Chi Minh City, Vietnam, e-mail: huynhvanvan@tdt.edu.vn

Received 26.12.2015. 
class of mismatched uncertain systems involving exogenous disturbances. In order to reduce conservative, the authors of [6] have presented a new observer-based stabilization for mismatched uncertain systems.

However, time delays are not included in the above approaches [3-6]. Time-delay systems often feature in real-world problems, for example, chemical processes, biological systems, economic systems, and hydraulic/pneumatic systems. Time delay commonly leads to a degradation and/or instability in system performance [1-2]. This has motivated the application of observer-based control to time delay systems, leading to many useful results [7-16].

In [7], observer design approach is proposed based on $H_{\infty}$ control theory. Based on communication disturbance observer, the practical design procedure of the time delayed control system was developed in [8]. In [9], a class of memoryless adaptive robust state observers is proposed for uncertain nonlinear time-delay systems. Based on Lyapunov stability theorem and LMI technique, an observer-based sliding mode control is developed for uncertain state-delayed systems [10]. The non-fragile observer and controller design method is presented in [11]. A repetitive control design is presented for timedelay systems via disturbance observer [12]. The authors of [13] have presented a new reduced-order observer to stabilize a class of nonlinear systems involving time-varying delay. The delay dependent stability and stabilization is proposed for systems with interval time-varying delay [14]. In [15], a nonlinear-observer-based controller is designed to stabilize a class of time delay system with triangular structure. The proposed observerbased stabilization conditions are developed for a class of matched uncertain system with both the input and the output delays [16]. In the above works there are obtained important results related to observer-based control, especially stability of time-delay systems is assured under certain conditions.

It is worth to point out that there are some limitations in existing design methods of observer-based control in application for the stability of mismatched uncertain timedelay systems. First, most of the existing results cannot be applied for time-delay systems with mismatched parameter uncertainties in the state matrix [7-11, 13-16]. Second, these obtained methods given in $[3-5,10,15]$ remain conservative due to using bilinear matrix inequalities (BMI) conditions. Therefore, it is important to develop a new observer-based control with a single-step approach to stabilize the uncertain time-delay systems with mismatched parameter uncertainties in the state matrix and in the delayed state matrix.

In this paper, we extend the concept of observer-based control, introduced by [3] and [6], for the aim of stabilizing mismatched matched time-delay systems. The main contributions of this paper are as follows:

- The mismatched uncertain time-delay systems investigated in this study include mismatched parameter uncertainties in the state matrix and in the delayed state matrix.

- The appropriate LMI stability condition by the Lyapunov method is derived to guarantee the stability of the mismatched uncertain time-delay system. The proposed LMI condition is solved without any additional restrictive conditions. 
- The controller gain matrix and the observer gain matrix are directly computed from the LMI formulation.

\section{Statement of the problem}

Consider the following mismatched uncertain systems with time-delay:

$$
\begin{aligned}
\dot{x}(t) & =[A+\Delta A] x(t)+\left[A_{d}+\Delta A_{d}\right] x(t-d)+B u(t) \\
y(t) & =C x(t)
\end{aligned}
$$

where $x(t) \in \mathfrak{R}^{n}$ is the vector of continuous-time state variables, $u(t) \in \mathfrak{R}^{m}$ is the vector of control inputs, $y(t) \in \mathfrak{R}^{p}$ is the system outputs. The symbols $x(t-d(t))$ and $y(t-d(t))$ represent delayed states and delayed outputs, respectively. The known function $d=d(t)$ is the time-varying delay which is assumed to be continuous, non-negative and bounded in $\Re_{+}$, that is, $\bar{d}:=\sup _{t \in \Re^{+}}\{d(t)\}<\infty$. The initial conditions for the system is given by $x(t)=\phi(t),(t \in[-\bar{d}, 0])$ where $\phi(t)$ is continuous in $[-\bar{d}, 0]$. The matrices $S, B, C$ and $A_{d}$ are constant matrices with appropriate dimensions. The terms $\Delta A$ and $\Delta A_{d}$ represent the mismatched uncertainties of the plant, which the matching conditions are not satisfied.

The following assumptions are useful for the development of our work.

Assumption 1. The matrix pair $(A, C)$ is observable.

Assumption 2. The mismatched parameter uncertainties in the state matrix are satisfied as $\Delta A=M F(t) N$, where $F(t)$ is unknown but bounded as $\|F(t)\| \leqslant 1$ and $M, N$ are known matrices of appropriate dimensions.

Assumption 3. The mismatched parameter uncertainties in the delayed state matrix are given as $\Delta A_{d}(t)=M_{d} F_{d}(t) N_{d}$, where $F_{d}\left(x(t), x_{d}, t\right)$ is unknown but bounded as $\left\|F_{d}(t)\right\| \leqslant 1$ and $M_{d}, N_{d}$ are any nonzero matrices of appropriate dimensions.

The observer-based controller we consider in this paper is under the form:

$$
\begin{gathered}
\dot{\hat{x}}(t)=A \hat{x}(t)+A_{d} \hat{x}(t-d)+B u(t)+L[y(t)-\hat{y}(t)], \\
\hat{y}(t)=C \hat{x}(t), \\
u(t)=-K \hat{x}(t)
\end{gathered}
$$

where $\hat{x}(t) \in \mathfrak{R}^{n}$ is the estimation of $x(t), \hat{y}(t) \in \mathfrak{R}^{p}$ is the observer output, $K \in \mathfrak{R}^{m \times n}$ is the controller gain, $L \in \mathfrak{R}^{n \times p}$ is the observer gain.

Let $e(t)=x(t)-\hat{x}(t)$ and from the equations (1) and (2)-(4), we have

$$
\left[\begin{array}{l}
\dot{x}(t) \\
\dot{e}(t)
\end{array}\right]=\left[\begin{array}{l}
A-B K+\Delta A B K \\
\Delta A A-L C
\end{array}\right]\left[\begin{array}{l}
x(t) \\
e(t)
\end{array}\right]+\left[\begin{array}{l}
A_{d}+\Delta A_{d} 0 \\
\Delta A_{d} A_{d}
\end{array}\right]\left[\begin{array}{l}
x(t-d) \\
e(t-d)
\end{array}\right] .
$$


Before ending this section, we would like to introduce the following lemmas, which will be used in the development of our main results

Lemma 6 [17] Let $X, Y$ and $F$ are real matrices of suitable dimension with $F^{T} F \leqslant I$ then, for any scalar $\varphi>0$, the following matrix inequality holds

$$
X F Y+Y^{T} F^{T} X^{T} \leqslant \varphi^{-1} X X^{T}+\varphi Y^{T} Y .
$$

Lemma 7 Assume that $x \in \mathfrak{R}^{n}, y \in \mathfrak{R}^{n}, N \in \mathfrak{R}^{n \times n}, N$ is a positive definite matrix. Then, the inequality

$$
x^{T} y+y^{T} x \leqslant \frac{1}{\varepsilon} x^{T} N^{-1} x+\varepsilon y^{T} N y
$$

holds for all $\varepsilon>0$.

Proof of lemma 2. For any $n \times n$ matrix $N>0, N^{\frac{1}{2}}$ is well defined and $N^{\frac{1}{2}}>0$. Let vector

$$
\vartheta=\sqrt{\frac{1}{\varepsilon}} N^{(-1 / 2)} x-\sqrt{\varepsilon} N^{1 / 2} y .
$$

Then, we have

$$
\begin{aligned}
& \vartheta^{T} \vartheta=\left(\sqrt{\frac{1}{\varepsilon}} N^{(-1 / 2)} x-\sqrt{\varepsilon} N^{1 / 2} y\right)^{T}\left(\sqrt{\frac{1}{\varepsilon}} N^{(-1 / 2)} x-\sqrt{\varepsilon} N^{1 / 2} y\right) \\
& =\left(\sqrt{\frac{1}{\varepsilon}} x^{T}\left(N^{-(1 / 2)}\right)^{T}-\sqrt{\varepsilon} y^{T}\left(N^{1 / 2}\right)^{T}\left(\sqrt{\frac{1}{\varepsilon}} N^{-(1 / 2)} x-\sqrt{\varepsilon} N^{1 / 2} y\right)\right. \\
& =\frac{1}{\varepsilon} x^{T} N^{-1} x-x^{T} y-y^{T} x+\varepsilon y^{T} N y .
\end{aligned}
$$

Since $\vartheta^{T} \vartheta \geqslant 0$, it is obvious that

$$
x^{T} y+y^{T} x \leqslant \frac{1}{\varepsilon} x^{T} N^{-1} x+\varepsilon y^{T} N y
$$

The proof is completed.

Lemma 8 [18] The following matrix inequality:

$$
\left[\begin{array}{cc}
Q(x) & \Pi(x) \\
\Pi(x)^{T} & R(x)
\end{array}\right]>0
$$

where $Q(x)=Q(x)^{T}, R(x)=R(x)^{T}$ and $\Pi(x)$ depend affinity on $x$, is equivalent to $R(x)>$ $0, Q(x)-\Pi(x) R(x)^{-1} \Pi(x)^{T}>0$. 


\section{Main results}

The following theorem holds:

Theorem 13 System (1) is robustly stabilizable by the observer-based control (2)-(4) if there exist four positive definite matrices $R \in \Re^{n \times n}, P \in \mathfrak{R}^{n \times n}, Q_{1} \in \mathfrak{R}^{n \times n}, Q_{2} \in \mathfrak{R}^{n \times n}$, two matrices $\hat{K} \in \Re^{m \times n}, \hat{L} \in \Re^{n \times p}$ and positive scalars $\varepsilon_{1}>0, \varepsilon_{2}>0, \varepsilon_{3}>0, \varepsilon_{4}>0$, $\varepsilon_{5}>0$, and $\varepsilon_{6}>0$ so that the following linear matrix inequality (LMI) condition (6) is feasible

$\left[\begin{array}{cccccccccccc}\Xi_{1} & 0 & R A_{d} & 0 & R B \hat{K} & 0 & -R B \hat{K} & I & 0 & R M_{d} & R M & 0 \\ 0 & \Xi_{2} & 0 & P A_{d} & 0 & I & 0 & 0 & P M_{d} & 0 & 0 & P M \\ A_{d}^{T} R & 0 & \Xi_{3} & 0 & 0 & 0 & 0 & 0 & 0 & 0 & 0 & 0 \\ 0 & A_{d}^{T} P & 0 & -Q_{2} & 0 & 0 & 0 & 0 & 0 & 0 & 0 & 0 \\ \hat{K}^{T} B^{T} R & 0 & 0 & 0 & -\varepsilon_{5} R & 0 & 0 & 0 & 0 & 0 & 0 & 0 \\ 0 & I & 0 & 0 & 0 & -\varepsilon_{5}^{-1} R & 0 & 0 & 0 & 0 & 0 & 0 \\ -\hat{K}^{T} B^{T} R & 0 & 0 & 0 & 0 & 0 & -\varepsilon_{6} R & 0 & 0 & 0 & 0 & 0 \\ I & 0 & 0 & 0 & 0 & 0 & 0 & -\varepsilon_{6}^{-1} R & 0 & 0 & 0 & 0 \\ 0 & M_{d}^{T} P & 0 & 0 & 0 & 0 & 0 & 0 & -\varepsilon_{4} I & 0 & 0 & 0 \\ M_{d}^{T} R & 0 & 0 & 0 & 0 & 0 & 0 & 0 & 0 & -\varepsilon_{3} I & 0 & 0 \\ M^{T} R & 0 & 0 & 0 & 0 & 0 & 0 & 0 & 0 & 0 & -\varepsilon_{2} I & 0 \\ 0 & M^{T} P & 0 & 0 & 0 & 0 & 0 & 0 & 0 & 0 & 0 & -\varepsilon_{1} I\end{array}\right]<0$

where $\Xi_{1}=R A+A^{T} R+Q_{1}+\varepsilon_{7} N^{T} N, \Xi_{2}=P A-\hat{L} C+A^{T} P-C^{T} \hat{L}^{T}+Q_{2}$ and $\Xi_{3}=$ $-Q_{1}+\varepsilon_{8} N_{d}^{T} N_{d}, \varepsilon_{7}=\frac{1}{\varepsilon_{1}}+\frac{1}{\varepsilon_{2}} \varepsilon_{8}=\frac{1}{\varepsilon_{3}}+\frac{1}{\varepsilon_{4}}$. Hence, the stabilizing observer-based control gains are given by $K=\hat{K} R^{-1}$ and $L=P^{-1} \hat{L}$.

Proof Take symmetric positive-definite matrix variables $P \in \mathfrak{R}^{n \times n}>0, R \in \mathfrak{R}^{n \times n}>0$, $Q_{1} \in \mathfrak{R}^{n \times n}>0$ and $Q_{2} \in \Re^{n \times n}>0$ and choose a Lyapunov functional as

$$
V(x(t), e(t))=\left[\begin{array}{l}
x(t) \\
e(t)
\end{array}\right]^{T}\left[\begin{array}{ll}
R & 0 \\
0 & P
\end{array}\right]\left[\begin{array}{l}
x(t) \\
e(t)
\end{array}\right]+\int_{t-\tau}^{t}\left[\begin{array}{l}
x(s) \\
e(s)
\end{array}\right]^{T}\left[\begin{array}{ll}
Q_{1} & 0 \\
0 & Q_{2}
\end{array}\right]\left[\begin{array}{l}
x(s) \\
e(s)
\end{array}\right] d s .
$$

Then, the time derivative of $V$ is given by

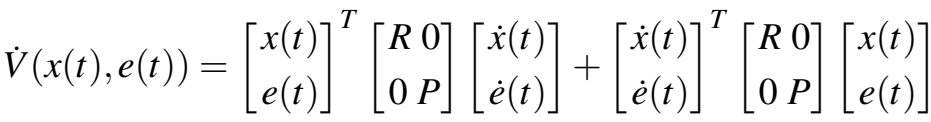

$$
\begin{aligned}
& +\left[\begin{array}{l}
x(t) \\
e(t)
\end{array}\right]^{T}\left[\begin{array}{ll}
Q_{1} & 0 \\
0 & Q_{2}
\end{array}\right]\left[\begin{array}{l}
x(t) \\
e(t)
\end{array}\right]-\left[\begin{array}{l}
x(t-d) \\
e(t-d)
\end{array}\right]^{T}\left[\begin{array}{ll}
Q_{1} & 0 \\
0 & Q_{2}
\end{array}\right]\left[\begin{array}{l}
x(t-d) \\
e(t-d)
\end{array}\right] .
\end{aligned}
$$


According to equation (5) and (8), we have

$$
\begin{aligned}
& \dot{V}(x(t), e(t))=\left[\begin{array}{l}
x(t) \\
e(t)
\end{array}\right]^{T}\left[\begin{array}{ll}
Q_{1} & 0 \\
0 & Q_{2}
\end{array}\right]\left[\begin{array}{l}
x(t) \\
e(t)
\end{array}\right]-\left[\begin{array}{l}
x(t-d) \\
e(t-d)
\end{array}\right]^{T}\left[\begin{array}{ll}
Q_{1} & 0 \\
0 & Q_{2}
\end{array}\right]\left[\begin{array}{l}
x(t-d) \\
e(t-d)
\end{array}\right] \\
& +\left[\begin{array}{c}
x(t) \\
e(t)
\end{array}\right]^{T}\left\{\left[\begin{array}{cc}
R(A-B K)+R \Delta A & R B K \\
P \Delta A & P(A-L C)
\end{array}\right]\right. \\
& \left.+\left[\begin{array}{cc}
(A-B K)^{T} R+(\Delta A)^{T} R & (\Delta A)^{T} P \\
(B K)^{T} R & (A-L C)^{T} P
\end{array}\right]\right\}\left[\begin{array}{l}
x(t) \\
e(t)
\end{array}\right] \\
& +\left[\begin{array}{l}
x(t) \\
e(t)
\end{array}\right]^{T}\left[\begin{array}{cc}
R A_{d}+R \Delta A_{d} & 0 \\
P \Delta A_{d} & P A_{d}
\end{array}\right]\left[\begin{array}{l}
x(t-d) \\
e(t-d)
\end{array}\right]+ \\
& {\left[\begin{array}{c}
x(t-d) \\
e(t-d)
\end{array}\right]^{T}\left[\begin{array}{cc}
A_{d}^{T} R+\left(\Delta A_{d}\right)^{T} R & \left(\Delta A_{d}\right)^{T} P \\
0 & A_{d}^{T} P
\end{array}\right]\left[\begin{array}{l}
x(t) \\
e(t)
\end{array}\right]} \\
& =\left[\begin{array}{c}
x(t) \\
e(t)
\end{array}\right]^{T}\left\{\begin{array}{cc}
R(A-B K)+(A-B K)^{T} R & R B K \\
(B K)^{T} R & P(A-L C)+(A-L C)^{T} P
\end{array}\right]+\left[\begin{array}{c}
0 \\
P M
\end{array}\right] F(t)\left[\begin{array}{ll}
N & 0
\end{array}\right] \\
& \left.+\left[\begin{array}{l}
N^{T} \\
0
\end{array}\right] F^{T}(t)\left[\begin{array}{ll}
0 & M^{T} P
\end{array}\right]+\left[\begin{array}{c}
R M \\
0
\end{array}\right] F(t)\left[\begin{array}{ll}
N & 0
\end{array}\right]+\left[\begin{array}{c}
N^{T} \\
0
\end{array}\right] F^{T}(t)\left[\begin{array}{ll}
M^{T} R & 0
\end{array}\right]\right\}\left[\begin{array}{l}
x(t) \\
e(t)
\end{array}\right] \\
& +\left[\begin{array}{c}
x(t) \\
e(t)
\end{array}\right]^{T}\left\{\left[\begin{array}{cc}
R A_{d} & 0 \\
0 & P A_{d}
\end{array}\right]+\left[\begin{array}{c}
R M_{d} \\
0
\end{array}\right] F_{d}(t)\left[\begin{array}{ll}
N_{d} & 0
\end{array}\right]+\left[\begin{array}{c}
0 \\
P M_{d}
\end{array}\right] F_{d}(t)\left[\begin{array}{ll}
N_{d} & 0
\end{array}\right]\right\}\left[\begin{array}{l}
x(t-d) \\
e(t-d)
\end{array}\right] \\
& +\left[\begin{array}{c}
x(t-d) \\
e(t-d)
\end{array}\right]^{T}\left\{\left[\begin{array}{cc}
A_{d}^{T} R & 0 \\
0 & A_{d}^{T} P
\end{array}\right]+\left[\begin{array}{c}
N_{d}^{T} \\
0
\end{array}\right] F_{d}^{T}(t)\left[\begin{array}{ll}
M_{d}^{T} R & 0
\end{array}\right]\right. \\
& \left.+\left[\begin{array}{c}
N_{d}^{T} \\
0
\end{array}\right] F_{d}^{T}(t)\left[\begin{array}{ll}
0 & M_{d}^{T} P
\end{array}\right]\right\}\left[\begin{array}{l}
x(t) \\
e(t)
\end{array}\right] \\
& +\left[\begin{array}{l}
x(t) \\
e(t)
\end{array}\right]^{T}\left[\begin{array}{ll}
Q_{1} & 0 \\
0 & Q_{2}
\end{array}\right]\left[\begin{array}{l}
x(t) \\
e(t)
\end{array}\right]-\left[\begin{array}{l}
x(t-d) \\
e(t-d)
\end{array}\right]^{T}\left[\begin{array}{ll}
Q_{1} & 0 \\
0 & Q_{2}
\end{array}\right]\left[\begin{array}{l}
x(t-d) \\
e(t-d)
\end{array}\right] .
\end{aligned}
$$

By Lemma 1, it follows from (9) that

$$
\dot{V}(x(t), e(t)) \leqslant\left[\begin{array}{c}
x(t) \\
e(t)
\end{array}\right]^{T}\left\{\begin{array}{cc}
R(A-B K)+(A-B K)^{T} R & R B K \\
(B K)^{T} R & P(A-L C)+(A-L C)^{T} P
\end{array}\right]
$$




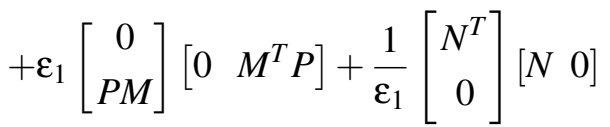

$$
\begin{aligned}
& \left.+\varepsilon_{2}\left[\begin{array}{c}
R M \\
0
\end{array}\right]\left[M^{T} R \quad 0\right]+\frac{1}{\varepsilon_{2}}\left[\begin{array}{c}
N^{T} \\
0
\end{array}\right]\left[\begin{array}{ll}
N & 0
\end{array}\right]\right\}\left[\begin{array}{l}
x(t) \\
e(t)
\end{array}\right] \\
& +\left[\begin{array}{l}
x(t) \\
e(t)
\end{array}\right]^{T}\left[\begin{array}{cc}
R A_{d} & 0 \\
0 & P A_{d}
\end{array}\right]\left[\begin{array}{l}
x(t-d) \\
e(t-d)
\end{array}\right]+\left[\begin{array}{c}
x(t-d) \\
e(t-d)
\end{array}\right]^{T}\left[\begin{array}{cc}
A_{d}^{T} R & 0 \\
0 & A_{d}^{T} P
\end{array}\right]\left[\begin{array}{l}
x(t) \\
e(t)
\end{array}\right]
\end{aligned}
$$

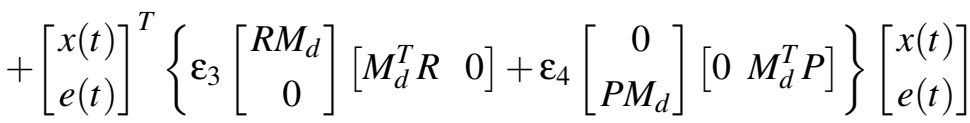

$$
\begin{aligned}
& +\left[\begin{array}{c}
x(t-d) \\
e(t-d)
\end{array}\right]^{T}\left\{\frac{1}{\varepsilon_{3}}\left[\begin{array}{r}
N_{d}^{T} \\
0
\end{array}\right]\left[\begin{array}{ll}
N_{d} & 0
\end{array}\right]+\frac{1}{\varepsilon_{4}}\left[\begin{array}{c}
N_{d}^{T} \\
0
\end{array}\right]\left[\begin{array}{ll}
N_{d} & 0
\end{array}\right]\right\}\left[\begin{array}{l}
x(t-d) \\
e(t-d)
\end{array}\right] \\
& +\left[\begin{array}{l}
x(t) \\
e(t)
\end{array}\right]^{T}\left[\begin{array}{ll}
Q_{1} & 0 \\
0 & Q_{2}
\end{array}\right]\left[\begin{array}{l}
x(t) \\
e(t)
\end{array}\right]-\left[\begin{array}{l}
x(t-d) \\
e(t-d)
\end{array}\right]^{T}\left[\begin{array}{ll}
Q_{1} & 0 \\
0 & Q_{2}
\end{array}\right]\left[\begin{array}{l}
x(t-d) \\
e(t-d)
\end{array}\right] \\
& =\left[\begin{array}{c}
x(t) \\
e(t)
\end{array}\right]^{T}\left\{\left[\begin{array}{cc}
R A+A^{T} R & 0 \\
0 & P(A-L C)+(A-L C)^{T} P
\end{array}\right]\right. \\
& +\left[\begin{array}{c}
R B K \\
0
\end{array}\right]\left[\begin{array}{ll}
0 & I
\end{array}\right]+\left[\begin{array}{l}
0 \\
I
\end{array}\right]\left[(B K)^{T} R \quad 0\right]+\left[\begin{array}{cc}
-R B K \\
0
\end{array}\right]\left[\begin{array}{ll}
I & 0
\end{array}\right]+\left[\begin{array}{l}
I \\
0
\end{array}\right]\left[-(B K)^{T} R \quad 0\right] \\
& +\varepsilon_{1}\left[\begin{array}{c}
0 \\
P M
\end{array}\right]\left[\begin{array}{ll}
0 & M^{T} P
\end{array}\right]+\frac{1}{\varepsilon_{1}}\left[\begin{array}{r}
N^{T} \\
0
\end{array}\right]\left[\begin{array}{ll}
N & 0
\end{array}\right] \\
& \left.+\varepsilon_{2}\left[\begin{array}{c}
R M \\
0
\end{array}\right]\left[M^{T} R \quad 0\right]+\frac{1}{\varepsilon_{2}}\left[\begin{array}{c}
N^{T} \\
0
\end{array}\right]\left[\begin{array}{ll}
N & 0
\end{array}\right]\right\}\left[\begin{array}{c}
x(t) \\
e(t)
\end{array}\right] \\
& +\left[\begin{array}{l}
x(t) \\
e(t)
\end{array}\right]^{T}\left[\begin{array}{l}
R A_{d} 0 \\
0 P A_{d}
\end{array}\right]\left[\begin{array}{l}
x(t-d) \\
e(t-d)
\end{array}\right]+\left[\begin{array}{l}
x(t-d) \\
e(t-d)
\end{array}\right]^{T}\left[\begin{array}{l}
A_{d}^{T} R 0 \\
0 A_{d}^{T} P
\end{array}\right]\left[\begin{array}{l}
x(t) \\
e(t)
\end{array}\right] \\
& +\left[\begin{array}{c}
x(t) \\
e(t)
\end{array}\right]^{T}\left\{\varepsilon_{3}\left[\begin{array}{c}
R M_{d} \\
0
\end{array}\right]\left[M_{d}^{T} R \quad 0\right]+\varepsilon_{4}\left[\begin{array}{c}
0 \\
P M_{d}
\end{array}\right]\left[\begin{array}{ll}
0 & M_{d}^{T} P
\end{array}\right]\right\}\left[\begin{array}{l}
x(t) \\
e(t)
\end{array}\right] \\
& +\left[\begin{array}{c}
x(t-d) \\
e(t-d)
\end{array}\right]^{T}\left\{\frac{1}{\varepsilon_{3}}\left[\begin{array}{r}
N_{d}^{T} \\
0
\end{array}\right]\left[\begin{array}{ll}
N_{d} & 0
\end{array}\right]+\frac{1}{\varepsilon_{4}}\left[\begin{array}{c}
N_{d}^{T} \\
0
\end{array}\right]\left[\begin{array}{ll}
N_{d} & 0
\end{array}\right]\right\}\left[\begin{array}{l}
x(t-d) \\
e(t-d)
\end{array}\right] \\
& +\left[\begin{array}{l}
x(t) \\
e(t)
\end{array}\right]^{T}\left[\begin{array}{ll}
Q_{1} & 0 \\
0 & Q_{2}
\end{array}\right]\left[\begin{array}{l}
x(t) \\
e(t)
\end{array}\right]-\left[\begin{array}{l}
x(t-d) \\
e(t-d)
\end{array}\right]^{T}\left[\begin{array}{ll}
Q_{1} & 0 \\
0 & Q_{2}
\end{array}\right]\left[\begin{array}{l}
x(t-d) \\
e(t-d)
\end{array}\right] .
\end{aligned}
$$

In addition, applying Lemma 2, we have 


$$
\begin{aligned}
& {\left[\begin{array}{c}
R B K \\
0
\end{array}\right]\left[\begin{array}{ll}
0 & I
\end{array}\right]+\left[\begin{array}{l}
0 \\
I
\end{array}\right]\left[(B K)^{T} R \quad 0\right] \leqslant \varepsilon_{5}\left[\begin{array}{c}
R B K \\
0
\end{array}\right] R\left[(B K)^{T} R \quad 0\right]+\frac{1}{\varepsilon_{5}}\left[\begin{array}{l}
0 \\
I
\end{array}\right] R^{-1}\left[\begin{array}{ll}
0 & I
\end{array}\right]} \\
& =\varepsilon_{5}\left[\begin{array}{c}
R B K R \\
0
\end{array}\right] R^{-1}\left[R(B K)^{T} R 0\right]+\frac{1}{\varepsilon_{5}}\left[\begin{array}{l}
0 \\
I
\end{array}\right] R^{-1}\left[\begin{array}{ll}
0 & I
\end{array}\right]
\end{aligned}
$$

and

$$
\begin{gathered}
{\left[\begin{array}{c}
-R B K \\
0
\end{array}\right]\left[\begin{array}{ll}
I & 0
\end{array}\right]+\left[\begin{array}{l}
I \\
0
\end{array}\right]\left[\begin{array}{ll}
-(B K)^{T} R & 0
\end{array}\right] \leqslant \varepsilon_{6}\left[\begin{array}{c}
-R B K \\
0
\end{array}\right] R\left[-(B K)^{T} R \quad 0\right]+\frac{1}{\varepsilon_{6}}\left[\begin{array}{l}
I \\
0
\end{array}\right] R^{-1}\left[\begin{array}{ll}
I & 0
\end{array}\right]} \\
=\varepsilon_{6}\left[\begin{array}{c}
-R B K R \\
0
\end{array}\right] R^{-1}\left[-R(B K)^{T} R \quad 0\right]+\frac{1}{\varepsilon_{6}}\left[\begin{array}{l}
I \\
0
\end{array}\right] R^{-1}\left[\begin{array}{ll}
I & 0
\end{array}\right] .
\end{gathered}
$$

According to equations (10), (11) and (12), we achieve

$$
\begin{aligned}
& \dot{V}(x(t), e(t)) \leqslant\left[\begin{array}{l}
x(t) \\
e(t)
\end{array}\right]^{T}\left\{\left[\begin{array}{cc}
R A+A^{T} R & 0 \\
0 & P(A-L C)+(A-L C)^{T} P
\end{array}\right]\right. \\
& +\varepsilon_{5}\left[\begin{array}{c}
R B K R \\
0
\end{array}\right] R^{-1}\left[R(B K)^{T} R \quad 0\right]+\frac{1}{\varepsilon_{5}}\left[\begin{array}{l}
0 \\
I
\end{array}\right] R^{-1}\left[\begin{array}{ll}
0 & I
\end{array}\right] \\
& +\varepsilon_{6}\left[\begin{array}{c}
-R B K R \\
0
\end{array}\right] R^{-1}\left[\begin{array}{ll}
-R(B K)^{T} R & 0
\end{array}\right]+\frac{1}{\varepsilon_{6}}\left[\begin{array}{l}
I \\
0
\end{array}\right] R^{-1}\left[\begin{array}{ll}
i & 0
\end{array}\right]
\end{aligned}
$$

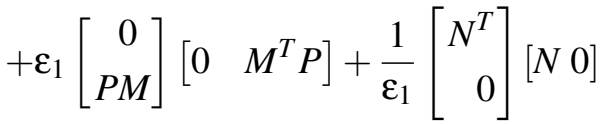

$$
\begin{aligned}
& \left.+\varepsilon_{2}\left[\begin{array}{c}
R M \\
0
\end{array}\right]\left[\begin{array}{ll}
M^{T} R & 0
\end{array}\right]+\frac{1}{\varepsilon_{2}}\left[\begin{array}{c}
N^{T} \\
0
\end{array}\right]\left[\begin{array}{ll}
N & 0
\end{array}\right]\right\}\left[\begin{array}{l}
x(t) \\
e(t)
\end{array}\right] \\
& +\left[\begin{array}{l}
x(t) \\
e(t)
\end{array}\right]^{T}\left[\begin{array}{cc}
R A_{d} & 0 \\
0 & P A_{d}
\end{array}\right]\left[\begin{array}{l}
x(t-d) \\
e(t-d)
\end{array}\right]+\left[\begin{array}{l}
x(t-d) \\
e(t-d)
\end{array}\right]^{T}\left[\begin{array}{cc}
A_{d}^{T} R & 0 \\
0 & A_{d}^{T} P
\end{array}\right]\left[\begin{array}{l}
x(t) \\
e(t)
\end{array}\right] \\
& +\left[\begin{array}{c}
x(t) \\
e(t)
\end{array}\right]^{T}\left\{\varepsilon_{3}\left[\begin{array}{c}
R M_{d} \\
0
\end{array}\right]\left[\begin{array}{ll}
M_{d}^{T} R & 0
\end{array}\right]+\varepsilon_{4}\left[\begin{array}{c}
0 \\
P M_{d}
\end{array}\right]\left[\begin{array}{ll}
0 & M_{d}^{T} P
\end{array}\right]\right\}\left[\begin{array}{l}
x(t) \\
e(t)
\end{array}\right] \\
& +\left[\begin{array}{c}
x(t-d) \\
e(t-d)
\end{array}\right]^{T}\left\{\frac{1}{\varepsilon_{3}}\left[\begin{array}{r}
N_{d}^{T} \\
0
\end{array}\right]\left[\begin{array}{ll}
N_{d} & 0
\end{array}\right]+\frac{1}{\varepsilon_{4}}\left[\begin{array}{c}
N_{d}^{T} \\
0
\end{array}\right]\left[\begin{array}{ll}
N_{d} & 0
\end{array}\right]\right\}\left[\begin{array}{l}
x(t-d) \\
e(t-d)
\end{array}\right] \\
& +\left[\begin{array}{l}
x(t) \\
e(t)
\end{array}\right]^{T}\left[\begin{array}{ll}
Q_{1} & 0 \\
0 & Q_{2}
\end{array}\right]\left[\begin{array}{l}
x(t) \\
e(t)
\end{array}\right]-\left[\begin{array}{l}
x(t-d) \\
e(t-d)
\end{array}\right]^{T}\left[\begin{array}{ll}
Q_{1} & 0 \\
0 & Q_{2}
\end{array}\right]\left[\begin{array}{l}
x(t-d) \\
e(t-d)
\end{array}\right]
\end{aligned}
$$


Define the augmented vector

$$
\chi(t)=\left[\begin{array}{llll}
x^{T}(t) & e^{T}(t) & x^{T}(t-d) & e^{T}(t-d)
\end{array}\right]^{T} .
$$

Then, we can get the following equation

$$
\dot{V}(x(t), e(t)) \leqslant \chi^{T}(t) \Omega \chi(t)
$$

where

$\Omega=\left[\begin{array}{l}{\left[\begin{array}{lr}R A+A^{T} R+\varepsilon_{7} N^{T} N+\varepsilon_{2} R M M^{T} R+\varepsilon_{3} R M_{d} M_{d}^{T} R+Q_{1} & 0 \\ 0 & P A-\hat{L} C+A^{T} P-C^{T} \hat{L}^{T}+\varepsilon_{1} P M M^{T} P+\varepsilon_{4} P M_{d} M_{d}^{T} P+Q_{2}\end{array}\right]+\Psi\left[\begin{array}{lr}R A_{d} & 0 \\ 0 & P A_{d}\end{array}\right]} \\ {\left[\begin{array}{ll}A_{d}^{T} R & 0 \\ 0 & A_{d}^{T} P\end{array}\right]} \\ -\left[\begin{array}{ll}Q_{1} & 0 \\ 0 & Q_{2}\end{array}\right]+\left[\begin{array}{rr}\varepsilon_{8} N_{d}^{T} N_{d} & 0 \\ 0 & 0\end{array}\right]\end{array}\right]$,

and

$$
\begin{gathered}
\Psi=\varepsilon_{5}\left[\begin{array}{c}
R B \hat{K} \\
0
\end{array}\right] R^{-1}\left[\hat{K}^{T} B^{T} R 0\right]+\frac{1}{\varepsilon_{5}}\left[\begin{array}{l}
0 \\
I
\end{array}\right] R^{-1}[0 I]+\varepsilon_{6}\left[\begin{array}{c}
-R B \hat{K} \\
0
\end{array}\right] R^{-1}\left[-\hat{K}^{T} B^{T} R 0\right]+\Theta \\
\Theta=\frac{1}{\varepsilon_{6}}\left[\begin{array}{l}
I \\
0
\end{array}\right] R^{-1}\left[\begin{array}{ll}
I & 0], \quad \hat{K}=K R, \quad \hat{L}=P L .
\end{array}\right.
\end{gathered}
$$

Applying lemma 3 to LMI (6), we obtain

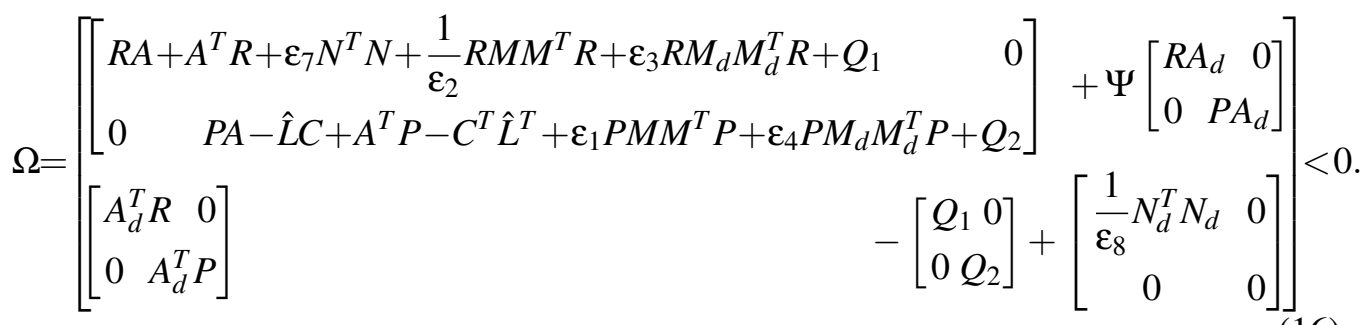

From equations (15) and (16), it is obvious that

$$
\dot{V}(x(t), e(t))<0 .
$$

The inequality (17) show that if LMI (6) holds, which further implies that the system (1) is robustly stabilizable by the observer-based control (2)-(4).

Remark 2 Compared to recent LMI methods [3-5, 10, 15], the proposed method offers less constraint in the LMI equation making it easier to find a feasible solution. 


\section{Numerical example}

In this section, to illustrate the effectiveness of the proposed observer-based control, the proposed method will be applied to the following mismatched uncertain time-delay system, which is modified from [10].

$$
\begin{gathered}
\dot{x}(t)=\left\{\left[\begin{array}{ccc}
-1.8 & 1 & 0 \\
0.6 & -1.5 & 0 \\
0 & 1 & -1
\end{array}\right]+\Delta A\right\} x(t) \\
+\left\{\left[\begin{array}{ccc}
0.2 & 0.1 & 0.1 \\
0.1 & 0.1 & 0 \\
0 & 0.1 & 0
\end{array}\right]+\Delta A_{d}\right\} x_{d}+\left[\begin{array}{ll}
2 & 0 \\
0 & 1 \\
1 & 1
\end{array}\right] u(t), \\
y(t)=\left[\begin{array}{lll}
1 & 5 & 2 \\
3 & 1 & 0
\end{array}\right] x(t)
\end{gathered}
$$

where $x(t)=\left[\begin{array}{lll}x_{1} & x_{2} & x_{3}\end{array}\right]^{T} \in \mathfrak{R}^{3}, u(t) \in \mathfrak{R}^{2}$ and $y=\left[\begin{array}{ll}y_{1} & y_{2}\end{array}\right]^{T} \in \mathfrak{R}^{2}$. The mismatched parameter uncertainties in the state matrix are $\Delta A=M F(t) N$ with $F(t)=\sin (t)$, $M=\left[\begin{array}{ccc}0.1 & 0 & 0.1 \\ 0 & 0.1 & 0.1 \\ 0 & 0.1 & 0.1\end{array}\right]$ and $N=\left[\begin{array}{ccc}0.1 & 0 & 0.1 \\ 0.1 & 0.1 & 0 \\ 0.1 & 0 & 0.1\end{array}\right]$. The mismatched parameter uncertainties in the delayed state matrix are $\Delta A_{d}=M_{d} F_{d}(t) N_{d}$ with $F_{d}(t)=\cos (t)$, $M_{d}=\left[\begin{array}{ccc}0.1 & 0.1 & 0.1 \\ 0.1 & 0.1 & 0.1 \\ 0.1 & 0.1 & 0.1\end{array}\right]$ and $N_{d}=\left[\begin{array}{ccc}0.1 & 0 & 0.1 \\ 0 & 0.1 & 0.1 \\ 0.1 & 0 & 0.1\end{array}\right]$. The design parameters are chosen as $\alpha_{1}=0.9, \alpha_{2}=0.4, \alpha_{3}=0.3, \alpha_{4}=0.9, \alpha_{5}=0.2$ and $\alpha_{6}=0.5$. Then, by solving the LMI (6), we have the feasible solution $R=\left[\begin{array}{ccc}14.87 & 1.20 & -6.37 \\ 1.20 & 19.23 & -9.00 \\ -6.37 & -9.00 & 11.59\end{array}\right]$, $P=\left[\begin{array}{ccc}18.05 & 2.87 & -7.51 \\ 2.87 & 21.23 & -5.15 \\ -7.51 & -5.15 & 15.36\end{array}\right], \quad Q_{1}=\left[\begin{array}{ccc}16.247 & -7.930 & -2.156 \\ -7.930 & 28.229 & -12.752 \\ -2.156 & -12.752 & 9.797\end{array}\right]$, $Q_{2}=\left[\begin{array}{ccc}28.35 & -8.19 & -7.83 \\ -8.19 & 30.22 & -10.51 \\ -7.83 & -10.51 & 15.14\end{array}\right], \hat{K}=\left[\begin{array}{ccc}0.06 & 0.06 & -0.07 \\ -0.13 & -0.17 & 0.26\end{array}\right]$, and $\hat{L}=\left[\begin{array}{cc}-0.196 & 0.54 \\ 0.316 & -0.43 \\ -0.062 & 0.31\end{array}\right]$. The matrix $\Sigma$ is selected to be $\Sigma=\left[\begin{array}{lll}1.0954 & 0.2191\end{array}\right]$ and 
$K_{2}=\Sigma P \Sigma^{T}=0.0758$. Hence, the stabilizing observer-based control gains are given by

$K=\hat{K} R^{-1}=\left[\begin{array}{ccc}0.002 & 0.001 & -0.004 \\ 0.002 & 0.003 & 0.027\end{array}\right]$ and $L=P^{-1} \hat{L}=\left[\begin{array}{cc}-0.016 & 0.049 \\ 0.015 & -0.017 \\ -0.006 & 0.038\end{array}\right]$.

The initial conditions for the above system are selected to be $x(0)=\left[\begin{array}{lll}1 & 1 & 1\end{array}\right]^{T}$ and $\hat{x}(0)=\left[\begin{array}{lll}0 & 0 & 0\end{array}\right]^{T}$. The time-varying delay $d(t)$ is chosen as $d(t)=0.1$. From Fig. 1 to Fig. 4 , it clearly shows that the proposed controller is effective in dealing with mismatched parameter uncertainties in the state matrix and in the delayed state matrix. It should be pointed out that the controller given in [10] can not be applied for the system (18)-(19) because mismatched parameter uncertainties in the state matrix and in the delayed state matrix are not satisfied the matching conditions.

\section{Conclusion}

In this paper, a new approach to design observer-based controllers for mismatched uncertain time-delay systems is proposed. First, the sufficient condition in terms of linear matrix inequality is derived such that the stability of mismatched uncertain time-delay systems and the asymptotic convergence properties for the estimation error are assured. Second, the control and observer gains are given from single LMI feasible solution. Thus the drawback of the bilinear matrix inequalities approach often encountered in the literature is removed and conservatism is reduced, and robustness is enhanced.

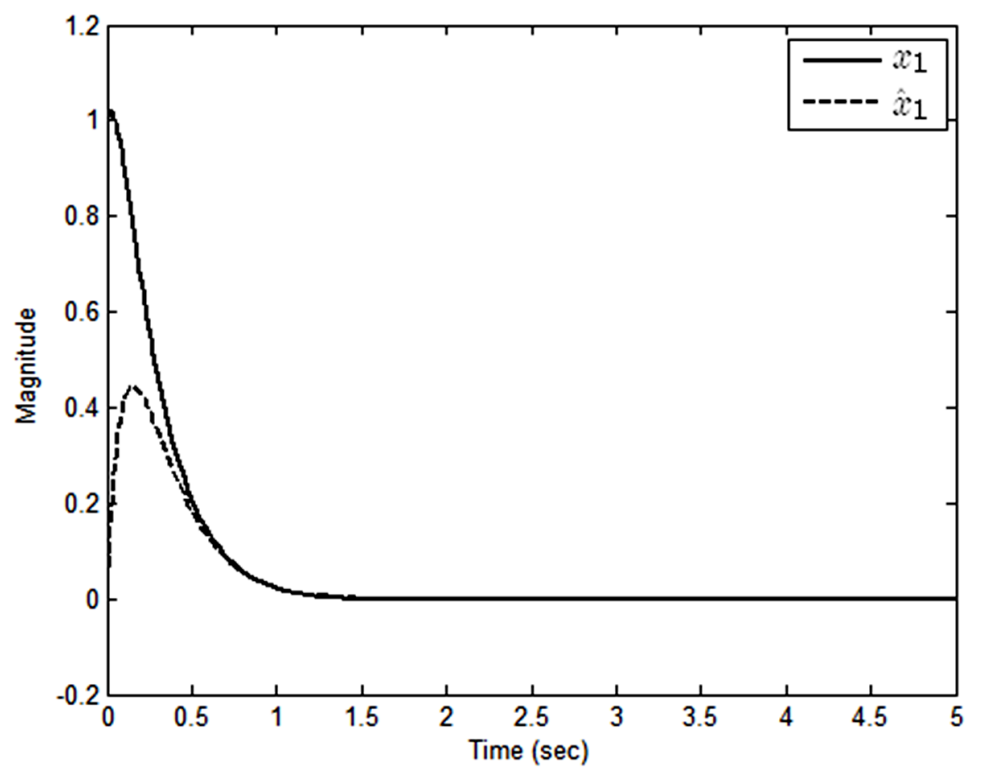

Figure 1: State $x_{1}$ and estimated state $\hat{x}_{1}$. 


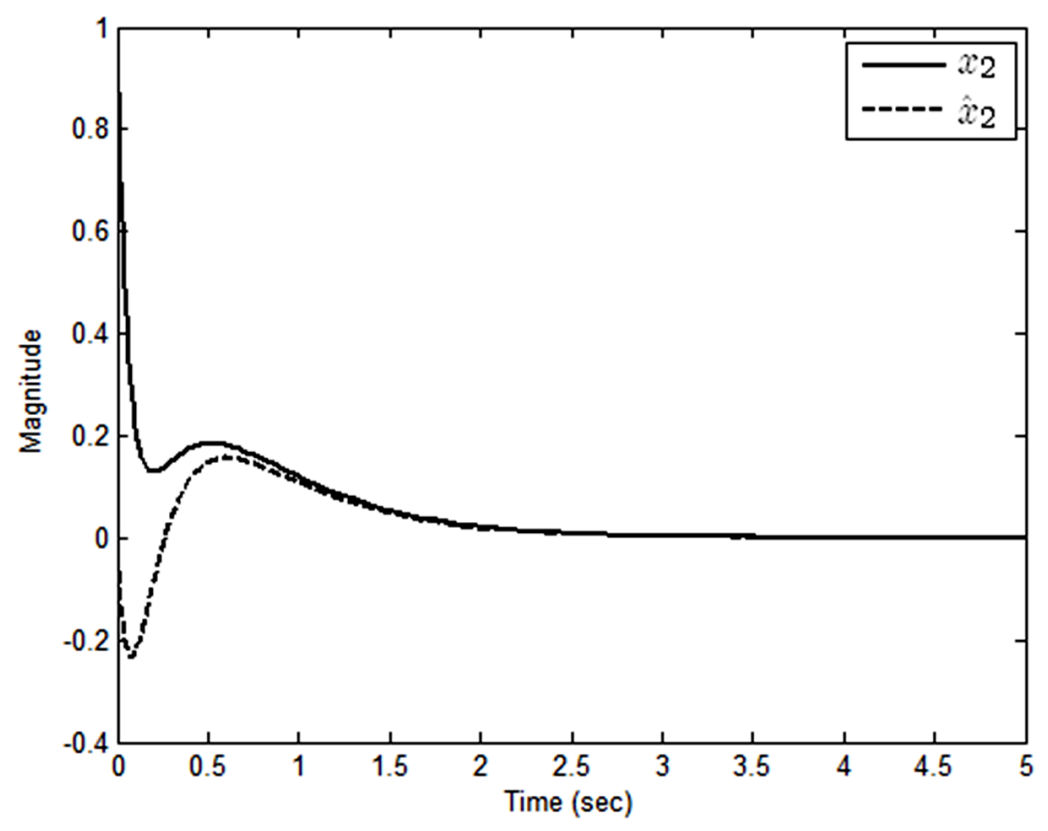

Figure 2: State $x_{2}$ and estimated state $\hat{x}_{2}$.

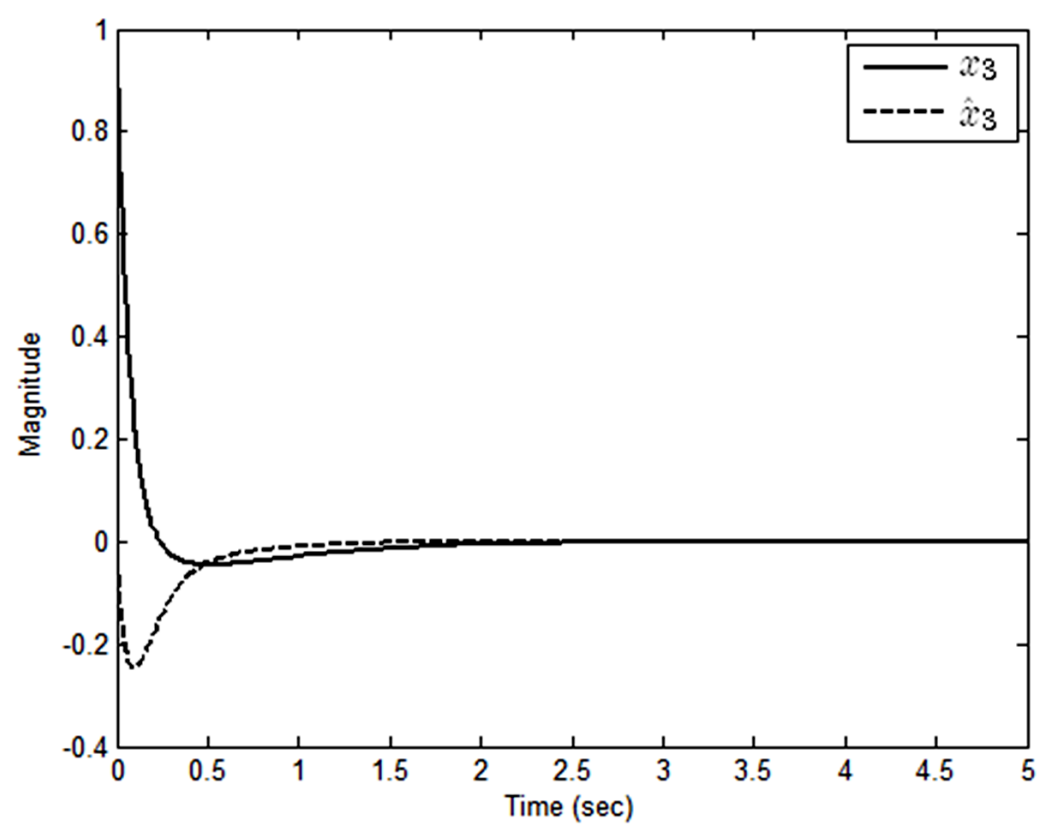

Figure 3: State $x_{3}$ and estimated state $\hat{x}_{3}$. 


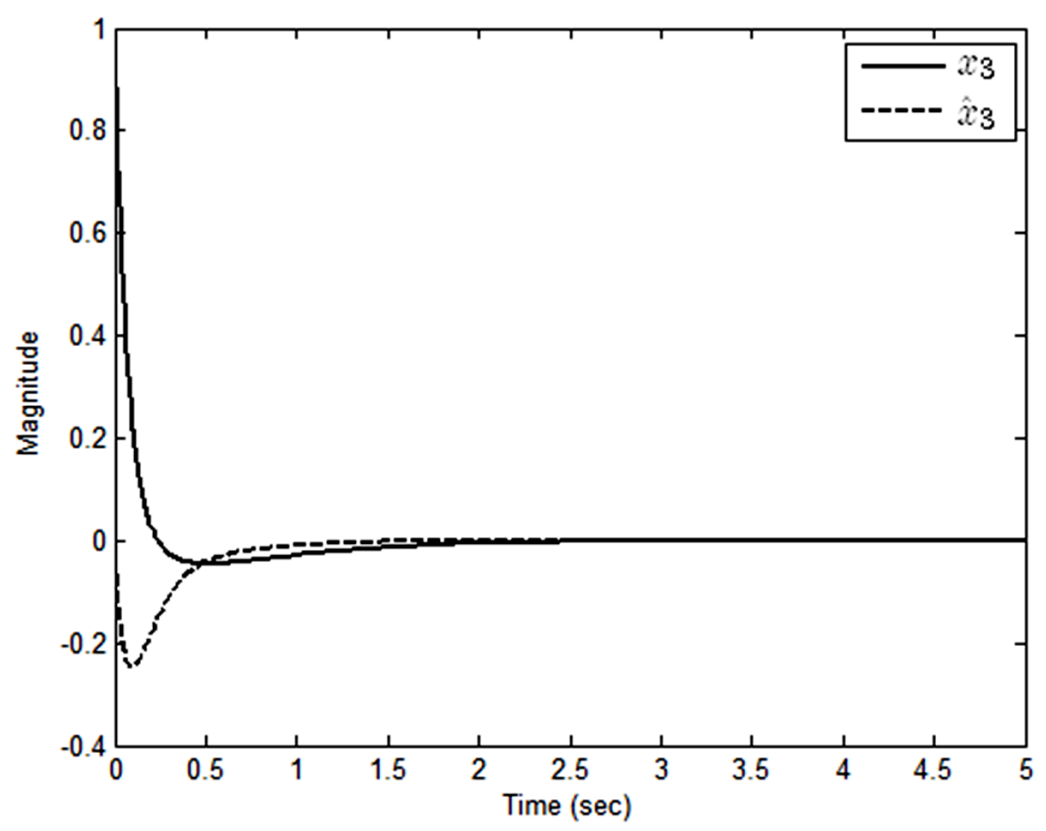

Figure 4: State estimation errors $e_{1}, e_{2}$ and $e_{3}$.

\section{References}

[1] X.G Yan, S. K. Spurgeon and C. Edwards: Static output feedback sliding mode control for time-varying delay systems with time-delayed nonlinear disturbances. Int. J. of Robust and Nonlinear Control, 20(7), (2010), 777-788.

[2] X.G. Yan, S. K. Spurgeon and C. Edwards: Memoryless static output feedback sliding mode control for nonlinear systems with delayed disturbances. IEEE Trans. on Automatic Control, 59(7), (2014), 1906-1912.

[3] C.H. LIEN: Robust observer-based control of systems with state perturbations via LMI approach. IEEE Trans. on Automatic Control, 49(8), (2004), 1365-1370.

[4] C.H. LIEN: An efficient method to design robust observer-based control of uncertain linear systems. Applied Mathematics and Computation, 158(1), (2004), 29-44.

[5] C.H LIEN and K.W YU: LMI optimization approach on robustness and $H_{\infty}$ control analysis for observer-based control of uncertain systems. Chaos, Solitons and Fractals, 36(3), (2008), 617-627.

[6] S. WANG and Y. JIANG: On LMI conditions to design observer-based controllers for linear systems with parameter uncertainties. Automatica, 49(12), (2013), 37003704 . 
[7] Y. FU , W. Di, P. ZANG and G. DuAN: Design of unknown input observer with $H_{\infty}$, performance for linear time-delay systems. J. of Systems Engineering and Electronics, 17(3), (2006), 606-610.

[8] K. NATORI: Stability analysis and practical design procedure of time delayed control systems with communication disturbance observer. IEEE Trans. on Industrial Informatics, 4(3), (2008), 185-197.

[9] H. WU: Memoryless adaptive robust asymptotic state observers for a class of nonlinear time-delay systems. IET Control Theory Applications, 3(7), (2009), 843-851.

[10] M.C PAI: Observer-based adaptive sliding mode control for nonlinear uncertain state-delayed systems. Int. J. of Control, Automation, and Systems, 7(4), (2009), 536-544.

[11] L. LI and Y. JIA: Observer-based resilient $L_{2}-L_{\infty}$ control for singular time-delay systems. IET Control Theory Applications, 3(10), (2009), 1351-1362.

[12] J. Na, R. Grino, R. Costa-Castello, X. Ren and Q. Chen: Repetitive controller for time-delay systems based on disturbance observer. IET Control Theory Applications, 4(11), (2010), 2391-2404.

[13] X.G YAN, K.S SARAH and C. EDWARD: Sliding mode control for time-varying delayed systems based on a reduced-order observer. Automatica, 46(8), (2010), 1354-1362.

[14] J. Zhang, Y. XiA, P. Shi and M.S. Mamoud: New results on stability and stabilisation of systems with interval time-varying delay. IET Control Theory Applications, 5(3), (2011), 429-436.

[15] I. SALIM: Observer-based control of a class of time-delay nonlinear systems having triangular structure. Automatica, 47(2), (2010), 388-394.

[16] R. Majeed, S. Admad, S and M. Rehan: Delay-range-dependent observerbased control of nonlinear systems under input and output time-delays. Applied Mathematics and Computation, 262 (2015), 145-159.

[17] J. ZHANG and Y. XIA: Design of static output feedback sliding mode control for uncertain linear systems. IEEE Trans. on Industrial Electronics, 57(6), (2010), 2161-2170.

[18] S. Boyd, E. L. Ghaoui, E. Feron and V. Balakrishna: Linear Matrix Inequalities in System and Control theory, SIAM, Philadelphia, 1998. 